\title{
Factors influencing the risk of recurrence in patients with esophageal carcinoma treated with surgery: A single institution analysis consisting of 1002 cases
}

\author{
YAPING XU ${ }^{1}$, QIXUN CHEN ${ }^{2}, \mathrm{XINMIN} \mathrm{YU}^{3}, \mathrm{XIMING} \mathrm{ZHOU}^{2}, \mathrm{XIAO} Z \mathrm{ZHENG}^{1}$ and WEIMIN MAO ${ }^{2}$ \\ Departments of ${ }^{1}$ Radiation Oncology, ${ }^{2}$ Thoracic Surgery and ${ }^{3}$ Medical Oncology, \\ Zhejiang Cancer Hospital, Hangzhou 310022, Zhejiang, P.R. China
}

Received July 17, 2012; Accepted October 18, 2012

DOI: $10.3892 / 01.2012 .1007$

\begin{abstract}
The aim of this study was to identify the risk of recurrence in patients with thoracic esophageal squamous cell carcinoma (TESCC) treated with curative surgery. The retrospective analysis included 1002 consecutive patients with TESCC who had been treated with curative surgery in Zhejiang Cancer Hospital, China, between 2003 and 2008. Univariate and multivariate analyses using the Kaplan-Meier method and Cox proportional hazards model, respectively, were performed to identify the independent risk factors for locoregional recurrence and all the recurrence events. The $2-$ and 5-year recurrence rates were 39.0 and 59.2\%, respectively. More than $85 \%$ of recurrences occurred within 36 months. The variables associated with a higher rate of recurrence in the univariate analysis were gender (male), length of tumor ( $\geq 5 \mathrm{~cm}$ ), depth of invasion (deeper), lymph node metastasis (greater), histological grade (higher) and vessel involvement (positive). By multivariate analyses, gender (HR, 1.7; 95\% CI, 1.2-2.5; $\mathrm{P}=0.002)$, depth of invasion (HR, 1.4; 95\% CI, 1.2-1.6; $\mathrm{P}<0.001)$ and lymph node involvement ( HR, 1.4; 95\% CI, 1.3-1.5; $\mathrm{P}<0.001)$ were independent predictive factors of recurrence. Post-operative radiotherapy or chemotherapy did not significantly prolong failure-free survival (FFS), particularly in patients with early-stage disease. Information regarding the depth of primary tumor invasion and the number of lymph nodes involved may help in evaluating the recurrence risk in patients with TESCC treated with curative surgery. Further studies are required to clarify the correlation between recurrence and the different multidisciplinary treatment approaches.
\end{abstract}

Correspondence to: Dr Weimin Mao, Department of Thoracic Surgery, Zhejiang Cancer Hospital, Hangzhou 310022, Zhejiang, P.R. China

E-mail: maowmzj1218@163.com

Key words: esophageal squamous cell carcinoma, surgical treatment, recurrence, post-operative adjuvant therapy

\section{Introduction}

Esophageal cancer (EC) is the eighth most common type of cancer worldwide. In China, EC is the fourth most common cause of mortality and is frequently located in the thorax, while $95 \%$ of EC is pathologically diagnosed as squamous cell carcinoma (1). Surgery, as with other thoracic malignancies $(2,3)$, is the preferred therapeutic strategy for EC patients. However, the majority of patients do not survive due to recurrence, even in the presence of radical resection and extended lymph node dissection. Numerous factors affect recurrence, including age, gender, tumor location, local tumor stage, degree of cell differentiation and the presence of lymph node metastases or vascular involvement (4-6). The knowledge of patterns of recurrence and its prevalence will be of great value in the clinic when designing therapeutic strategies, as it would provide evidence for determination of the extent of surgical resection and guide effective post-operative adjuvant therapy.

The aim of the present study was to determine the factors that influence the risk of recurrence in patients with thoracic esophageal squamous cell carcinoma (TESCC) that have been treated with curative surgery. The current study represents one of the largest analyses of patterns of failure of patients undergoing curative surgery for TESCC in Asia.

\section{Patients and methods}

Patients. From 2002-2008, patients with stage T1-4N0-3M0 EC who had each received an esophagectomy were recruited for the present study. Patients who had received pre-operative chemotherapy and/or radiotherapy were excluded. In addition, only patients who survived for $>3$ months following surgery were included in the present study in favor of post-operative adjuvant therapy, as certain patients undergoing surgery alone may not have survived the perioperative period prior to adjuvant therapy. Thus, patients treated with an esophagectomy with or without adjuvant therapy were enrolled. The study was approved by the ethics committee of Zhejiang Cancer Hospital, Hangzhou, China. Informed consent was obtained from the patients.

Assessment of recurrence. Patterns of failure were assessed by means of follow-up data. Following completion of treat- 
Table I. Patient characteristics $(n=1002)$.

\begin{tabular}{|c|c|c|}
\hline Characteristic & No. patients & $\%$ \\
\hline \multicolumn{3}{|l|}{ Gender } \\
\hline Male & 894 & 89 \\
\hline Female & 108 & 11 \\
\hline \multicolumn{3}{|c|}{ Length of tumor (cm) } \\
\hline$<5$ & 568 & 57 \\
\hline$\geq 5$ & 434 & 43 \\
\hline \multicolumn{3}{|c|}{ Depth of invasion } \\
\hline pT1 & 100 & 10 \\
\hline pT2 & 170 & 17 \\
\hline pT3 & 669 & 67 \\
\hline pT4 & 63 & 6 \\
\hline \multicolumn{3}{|c|}{ Lymph node metastasis } \\
\hline pNO & 426 & 43 \\
\hline pN1 & 289 & 29 \\
\hline $\mathrm{pN} 2$ & 192 & 19 \\
\hline pN3 & 95 & 9 \\
\hline \multicolumn{3}{|c|}{ Histologic grade ${ }^{a}$} \\
\hline Well SCC & 200 & 20 \\
\hline Mod. SCC & 613 & 61 \\
\hline Poor SCC & 189 & 19 \\
\hline \multicolumn{3}{|c|}{ Vessel involvement } \\
\hline No & 862 & 86 \\
\hline Yes & 140 & 14 \\
\hline
\end{tabular}

aWell-, mod. (moderately) and poorly differentiated squamous cell carcinomas (SCC). The median patient age was 58 years (range, 36-86).

ment, the patients were surveyed every 3-4 months by physical examination, a chest computed tomography (CT) scan, and abdominal and cervical ultrasonography until tumor recurrence was evident. A bone scintigraphy was conducted if a patient presented with persistent localized bone pain. If tumor recurrence was identified, the same examinations were performed within 1 month and all recurrent tumors identified were regarded as the first recurrent tumor. All patients underwent routine post-surgical surveillance. The follow-up was completed in August, 2010.

The mode of tumor recurrence was classified as 'local' if it was within the surgical field and 'distant' when it was outside it. The former includes regional nodes and the surgical margin. The latter includes hematogenic metastasis (liver, lung, bone, skin, brain and adrenal), dissemination (pleural and peritoneal) and distant nodes (cervical, abdominal para-aortic and others).

Staging. Tumor size and extent was primarily coded according to the operative medical record and pathological findings. The number of lymph node metastases was determined based on pathological findings. This information was used for tumor node metastasis (TNM) classification according to the American Joint Committee on Cancer (AJCC) classification system (7th edition) (7).
Treatment. All patients were treated with radical resection. The standard surgical approach consisted of a limited thoracotomy on the right side and intrathoracic gastric tube reconstruction (the Ivor Lewis procedure) for lesions at the middle/lower third of the esophagus. Upper third lesions were treated by cervical anastomosis (the McKeown procedure). The majority of patients underwent two-field lymphadenectomy. The number of lymph nodes harvested per case ranged from 6-96 (median 28). Pyloroplasty and feeding jejunostomy were not routinely conducted. A nasogastric tube was inserted for each patient until the anastomotic wound had closed, as assessed by an esophagography on post-operative day 14

In the present study, as the role of post-operative adjuvant radiotherapy and/or chemotherapy in the treatment of ESCC was controversial at the time of treatment for these patients, post-operative adjuvant therapy was not mandatory. In each case, the utilization of post-operative adjuvant radiotherapy and/or chemotherapy was according to the physician's preference and the general physical condition of the patient. Cisplatin and 5-fluorouracil were the most frequently used agents in post-operative chemotherapy, although several other chemotherapeutic agents were also used. Post-operative adjuvant radiotherapy, if administered, was initiated 4-5 weeks postsurgery. A large T-shaped field encompassing the bilateral supraclavicular fossa, mediastinum and tumor bed was used. Radiation was initially administered through the anteroposterior field to $36 \mathrm{~Gy}$ at 2 Gy per fraction, then through the parallel opposing oblique fields to $14 \mathrm{~Gy}$, in order to avoid the spinal cord. Ten MV photons were used to deliver the radiation to the mediastinum through the anteroposterior and oblique fields. In all cases, the radiation dose was prescribed to the isocenter. The bilateral supraclavicular fossas were treated with 9-12 MeV electrons. In certain cases, targets were reduced on the basis of the patient's condition or the physician's judgment.

Statistical analyses. Survival and recurrence curves were estimated using the Kaplan-Meier method and compared using the log-rank test. Multivariate analyses were performed by the Cox regression model. The variables included two demographic variables (gender and age), two well-known prognostic factors (depth of tumor invasion and nodal involvement), extent of nodal dissection, length of tumor, histological grade, vessel involvement and post-operative adjuvant therapy. In total, 1002 patients underwent curative surgery (R0) for TESCC. Fatalities due to causes other than TESCC were considered censored observations at the time of death. The $\chi^{2}$ test was used to compare the frequencies of different ordinal data. Statistical analyses were performed using the Statistical Package for the Social Sciences (SPSS) software, version 13.0 (SPSS Inc., Chicago, IL, USA). All probability values were two-sided and $\mathrm{P}<0.05$ was considered to indicate a statistically significant difference.

\section{Results}

General data. In total, 1002 patients were investigated. The median follow-up period for the surviving patients was 51 months (range, 3-93 months). Patient characteristics and surgical/pathological details are provided in Table I. The sample consisted of 894 males and 108 females (median age, 
Table II. Recurrence according to the clinical characteristics of the 1002 patients.

\begin{tabular}{|c|c|c|c|c|c|c|}
\hline \multirow[b]{2}{*}{ Characteristic } & \multirow[b]{2}{*}{$\begin{array}{l}\text { No. of } \\
\text { patients }\end{array}$} & \multicolumn{3}{|c|}{ Recurrence } & \multirow[b]{2}{*}{$\chi^{2}$} & \multirow[b]{2}{*}{ P-value } \\
\hline & & $\begin{array}{l}\text { Median time } \\
\quad \text { (months) }\end{array}$ & 2 -year rate $(\%)$ & 5 -year rate $(\%)$ & & \\
\hline Gender & & & & & 17.794 & $<0.001$ \\
\hline Male & $485 / 894$ & 34.3 & 41.3 & 61.9 & & \\
\hline Female & $33 / 108$ & - & 21.5 & 39.2 & & \\
\hline Age (years) & & & & & 0.008 & 0.927 \\
\hline$<65$ & $391 / 757$ & 40.3 & 39.4 & 59.5 & & \\
\hline$\geq 65$ & $127 / 245$ & 33.3 & 38.3 & 58.3 & & \\
\hline Extent of nodal dissection & & & & & 0.607 & 0.738 \\
\hline Three-field & $76 / 139$ & 34.3 & 40.7 & 63.2 & & \\
\hline Two-field & $426 / 829$ & 37.3 & 39.2 & 58.5 & & \\
\hline Other & $16 / 34$ & 55.1 & 35.7 & 57.7 & & \\
\hline Length of tumor (cm) & & & & & 22.553 & $<0.001$ \\
\hline$<5$ & $258 / 568$ & 53.9 & 34.0 & 53.5 & & \\
\hline$\geq 5$ & $260 / 434$ & 26.5 & 45.8 & 66.7 & & \\
\hline Depth of invasion & & & & & 50.279 & $<0.001$ \\
\hline pT1 & $24 / 100$ & - & 13.3 & 39.4 & & \\
\hline pT2 & $68 / 170$ & & 27.7 & 49.2 & & \\
\hline pT3 & $386 / 669$ & 28.6 & 44.2 & 63.9 & & \\
\hline pT4 & $40 / 63$ & 23.1 & 56.7 & 77.3 & & \\
\hline Lymph node metastasis & & & & & 203.403 & $<0.001$ \\
\hline pNO & $153 / 426$ & - & 23.9 & 43.7 & & \\
\hline $\mathrm{pN} 1$ & $179 / 289$ & 16.9 & 61.5 & 78.9 & & \\
\hline $\mathrm{pN} 2$ & $121 / 192$ & 14.9 & 63.2 & 87.1 & & \\
\hline $\mathrm{pN} 3$ & $65 / 95$ & 11.9 & 77.0 & 96.3 & & \\
\hline Histologic grade $^{\mathrm{b}}$ & & & & & 18.124 & $<0.001$ \\
\hline Well SCC & $91 / 200$ & 54.3 & 34.8 & 56.5 & & \\
\hline Mod. SCC & $306 / 613$ & 41.5 & 37.5 & 56.8 & & \\
\hline Poor SCC & $121 / 189$ & 24.1 & 49.7 & 71.2 & & \\
\hline Vessel involvement & & & & & 24.988 & $<0.001$ \\
\hline No & $421 / 862$ & 43.4 & 36.3 & 56.4 & & \\
\hline Yes & $97 / 140$ & 20.3 & 55.6 & 75.7 & & \\
\hline
\end{tabular}

${ }^{\mathrm{a}}$ For females, T1, T2 and N0 median recurrence times were beyond the observation period. ${ }^{\mathrm{b}} \mathrm{Well}-$, mod (moderately) and poorly differentiated squamous cell carcinoma (SCC). The median patient age was 58 years (range, 36-86).

58 years; range, $36-86$ years). The majority of patients presented with stage T3 disease $(67 \%, 669 / 1002)$. The majority of tumors $(61 \%, 613 / 1002)$ were moderately differentiated SCC with no vessel involvement $(86 \%, 862 / 1002)$. Among the 1002 patients, 518 patients presented with lymph node metastasis. The average number of dissected lymph nodes was $28.2 \pm 14.4$ (mean \pm SD) nodes per case (median, 24; range, 2-96). The mean number of metastatic nodes was $2.1 \pm 0.8$ (median, 1 ; range, $0-36$ ) in the overall series and 4.1 \pm 1.9 (median, 2 ; range, $1-36$ ) in the pathologically lymph node-positive patients. A total of 239 patients received post-operative adjuvant chemotherapy ( $>2$ cycles); cisplatine and 5-fluorouracil were used most frequently (63\%). Post-operative adjuvant radiotherapy was administered to 255 patients. Males, and those aged $<65$ years, or with a tumor size $>5 \mathrm{~cm}$ or exhibiting a greater amount of lymph node metastasis, were more likely to receive post-operative adjuvant chemotherapy and/or radiotherapy.

Recurrence. Almost 50\% of recurrences were confirmed with a biopsy, and the remaining recurrences were scored using imaging studies. The data regarding survival and time to recurrence were available for all patients. At the time of analysis, 545 of the 1002 patients had survived, 484 of which were disease-free. Tumor recurrence was observed in 518 patients with a median follow-up time of 51 months. Locoregional and distant recurrence were observed in 49 and $44 \%$ of patients, respectively; while both locoregional and distant recurrence were present in $27 \%$ of patients. The median time to recur- 
Table III. Multivariate analysis of predictors for tumor recurrence.

\begin{tabular}{lcrr}
\hline Characteristic & Hazard ratio & 95\% confidence interval & P-value $^{\mathrm{a}}$ \\
\hline Gender & 1.732 & $1.214-2.471$ & 0.002 \\
Depth of invasion & 1.353 & $1.181-1.550$ & $<0.001$ \\
Lymph node metastasis & 1.420 & $1.319-1.528$ & $<0.001$
\end{tabular}

ap-values from Cox proportional hazards multivariate analysis.

Table IV. Stratified analysis according to T and N stages for different post-operative adjuvent therapies.

\begin{tabular}{|c|c|c|c|c|c|c|}
\hline \multirow[b]{2}{*}{ Characteristic } & \multicolumn{2}{|c|}{ Median recurrence-free time (months) } & \multirow[b]{2}{*}{ P-value } & \multicolumn{2}{|c|}{ Median recurrence-free time (months) } & \multirow[b]{2}{*}{ P-value } \\
\hline & No post-op. RT & Post-op. RT & & No post-op. CT & Post-op CT & \\
\hline \multicolumn{7}{|c|}{ Depth of invasion } \\
\hline pT1 & - & 52.7 & 0.121 & - & - & 0.049 \\
\hline pT2 & - & 30.2 & $<0.001$ & - & 30.1 & $<0.001$ \\
\hline pT3 & 27.3 & 24.5 & 0.278 & 28.7 & 23.1 & 0.355 \\
\hline pT4 & 15.7 & 18.7 & 0.692 & 16.3 & 17.6 & 0.887 \\
\hline \multicolumn{7}{|l|}{ LN metastais } \\
\hline $\mathrm{pNO}$ & - & 27.4 & $<0.001$ & - & 24.1 & $<0.001$ \\
\hline $\mathrm{pN} 1$ & 41.0 & 25.4 & 0.002 & 41.2 & 25.2 & 0.003 \\
\hline pN2 & 16.2 & 15.9 & 0.360 & 17.1 & 13.4 & 0.449 \\
\hline $\mathrm{pN} 3$ & 11.0 & 12.8 & 0.072 & 9.8 & 12.8 & 0.094 \\
\hline
\end{tabular}

${ }^{\text {aF }}$ or females, T1, T2 and N0 median recurrence time was beyond the observation period. Post-op., post-operative; RT, radiotherapy; CT, chemotherapy; LN, lymph node.

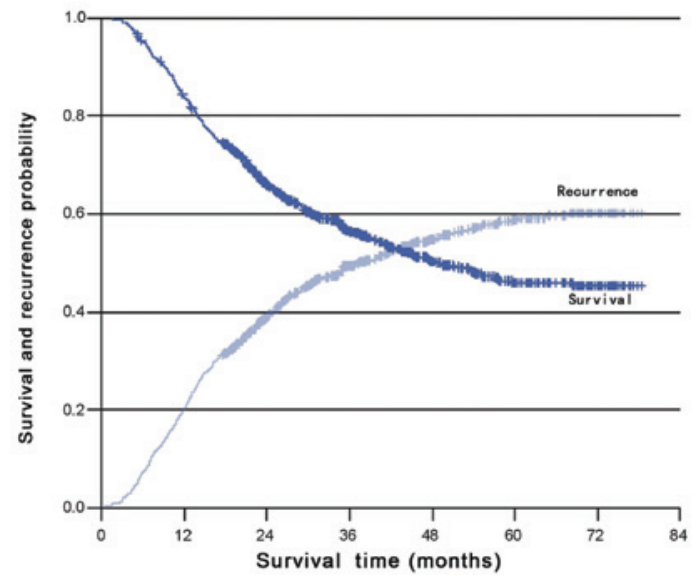

Figure 1. Kaplan-Meier estimates of survival and recurrence probability in the 1002 patients who underwent curative surgery (R0).

rence was 37.3 months (95\% CI, 31.1-43.6) and the cumulative rates of recurrence at 2 and 5 years were 39.0 and $59.2 \%$, respectively (Fig. 1). More than $85 \%$ of recurrences occurred within 36 months. Less than $10 \%$ of patients with $>10$ metastatic nodes were free from relapse 3 years post-surgery.

Univariate and multivariate analyses. Variables associated with a higher rate of recurrence in the univariate analysis were male gender $(\mathrm{P}<0.001)$, length of tumor $\geq 5 \mathrm{~cm}(\mathrm{P}<0.001)$, deeper depth of invasion $(\mathrm{P}<.001)$, greater lymph node metastasis $(\mathrm{P}<0.001)$, higher histological grade $(\mathrm{P}<0.001)$ and positive vessel involvement $(\mathrm{P}<0.001)$ (Table II). No correlation was observed between recurrence and the extent of lymph node dissection.

The multivariate analyses for recurrence demonstrated that gender (HR, 1.7; 95\% CI, 1.2-2.5; P=0.002), depth of invasion (HR, 1.4; 95\% CI, 1.2-1.6; $\mathrm{P}<0.001)$ and lymph node involvement (HR, 1.4; 95\% CI, 1.3-1.5; $\mathrm{P}<0.001)$ were independent predictive factors for recurrence. Male gender, deeper depth of primary tumor invasion and greater lymph node metastasis predicted an increase of recurrence (Table III).

Rate of recurrence in relation to post-operative adjuvant therapy. The rate of recurrence in relation to post-operative adjuvant therapy (including post-operative radiotherapy and/or post-operative chemotherapy) was also studied in detail. Postoperative radiotherapy was administered to 255 patients, while 239 patients received post-operative chemotherapy. Post-operative radiotherapy and/or chemotherapy did not significantly prolong failure-free survival (FFS), particularly in patients with early-stage disease (Table IV). In patients with pT3-4 disease, post-operative adjuvant radiotherapy and/or chemotherapy had a similar median recurrence-free time compared with surgery alone. However, in patients with 
pT1-2 disease, the post-operative adjuvant radiotherapy and/or chemotherapy arms had a shorter median recurrence-free time. For patients with $\mathrm{pN} 3$, the median recurrence-free time was prolonged by $\sim 2$ months following post-operative radiotherapy ( $\mathrm{P}=0.072$ ) and by $\sim 3$ months following post-operative chemotherapy $(\mathrm{P}=0.094)$; however, the difference was not significant.

\section{Discussion}

Esophageal cancer is a highly lethal disease. Despite best efforts, the 5-year survival rate following curative surgery rarely exceeds $30 \%$. Even patients with early-stage disease are at a relatively high risk of disease recurrence. A large proportion of patients do not survive due to disease recurrence either at the surgical site or at extrathoracic sites. The overall recurrence rate following curative surgery ranges from $34-79 \%$, while the locoregional, distant and both locoregional and distant recurrence rates range from 21-68, 18-63 and $5-47 \%$, respectively $(4,5,8-10)$. In the present study, the rate of tumor recurrence was similar to the findings mentioned previously in such a Chinese TESCC population treated with surgery. The present study also demonstrated that lymph node involvement and depth of tumor invasion are both significant predictive indicators of tumor recurrence; greater lymph node metastasis and deeper depth of invasion may suggest a greater tumor burden or a more aggressive tumor biology, and so the likelihood of locoregional or systemic recurrence may be speculated to be higher.

Surgical resection with radical esophagectomy and lymphadenectomy is currently the only well-established curative treatment methodology for patients with resectable ESCC when the patient is fit to undergo major surgery. Due to longitudinal lymphatic drainage through the extensive submucosal plexus, lymph node metastases may occur relatively early in all three body compartments (the abdomen, chest and neck), regardless of the location of the primary tumor. Matsubara et al (11) and Altorki et al (12) revealed a $30 \%$ incidence of pre-operatively unsuspected cervical nodal metastases, irrespective of the stage of the tumor, implying that cervical dissemination may be an early event. Therefore, certain surgeons perform a formal cervical lymphadenectomy as well as a two-field lymphadenectomy (abdomen and chest). Notably, a three-field lymphadenectomy achieves a higher rate of $\mathrm{R} 0$ resections, which may also lead to an improved oncological outcome. Cancer recurrence originates from micro-residues of cancer cells at the local or other sites. The surgical outcome may be improved in the case of an extended lymphadenectomy in patients with ESCC; however, there is no conclusive evidence thus far. Shiozaki et al (13) demonstrated that the prognosis of patients with recurrent nerve chain node metastasis was significantly better in a three-field dissection group than in a two-field dissection group. In our institution, the Ivor-Lewis approach with a two-field lymphadenectomy (extended mediastinal lymphadenectomy and lymph node dissection of the upper abdominal compartment) was the most frequently performed procedure $(82.7 \%)$. Our results indicated that tumor recurrence following extensive three-field dissection was similar to that following the less extensive two-field dissection $(\mathrm{P}=0.738)$. However, in our study the number of patients who received a three-field lymphadenectomy was only
139; the limited number of events made it difficult to evaluate recurrence differences. Therefore, a prospective observational study is required. Extended esophagectomy with three-field lymphadenectomy in treating operable TESCC has been demonstrated with a large number of patients in China (14). We may observe a recurrence of the three-field lymphadenectomy in this group of patients.

Another concern with this type of study is the multidisciplinary treatment approaches. An accurate assessment of factors influencing failure post-surgery may guide postoperative adjuvant therapy. Given the recognized risk of local recurrence, even in patients with early-stage disease, we advise a re-analysis of post-operative radiotherapy and chemotherapy. The role of adjuvant therapy of ESCC has been addressed in a number of clinical trials; however, until presently, whether post-operative radiotherapy and/or chemotherapy affect the therapeutic outcomes has remained controversial (15-20). Zhang et al (21) conducted a meta-analysis comprising a total of 1000 patients with esophageal cancer (ESCC and adenocarcinoma) in 2008. The results indicated that adjuvant chemotherapy did not significantly improve survival. Only the patients with pathologically positive lymph nodes demonstrated a positive trend towards improved survival, but this was not significant (OR, 0.76; 95\% CI, 0.538-1.083). Lu et al conducted a meta-analysis to study the clinical value of prophylactic radiotherapy for esophageal carcinoma after curative resection. The randomized trials did not demonstrate an influence of prophylactic radiotherapy on ESCC following curative resection (5-year survival OR, 1.26; 95\% CI, 0.687-2.290). However, patients with lymph node involvement had a statistically significant survival benefit 5 years after prophylactic radiotherapy, compared with surgery alone (5-year survival OR, 2.20; 95\% CI, 1.331-3.632) (22). In the present study, post-operative adjuvant radiotherapy and/or chemotherapy did not prolong FFS significantly in the whole study population. However, for patients with pT3-4 or pN3, the median recurrence-free time marginally increased following post-operative radiotherapy and/or chemotherapy (Table IV). Adjuvant radiotherapy and chemotherapy are theoretically capable of treating microscopic disease that remains following an incomplete surgical procedure and of increasing local control. However, caution should be taken in practice, as there is a lack of evidence in support of post-operative adjuvant therapy. Recently, Macdonald et al indicated that post-operative chemoradiation therapy significantly improved overall survival and relapse-free survival for patients at a high risk of recurrence of adenocarcinoma of the stomach or GE junction (23); however, the combination with the best outcome for ESCC is yet to be defined. The high frequency of recurrence and the short survival time following relapse are indicative of the need for aggressive initial treatment.

However, although almost $50 \%$ of recurrences were confirmed with a biopsy in this study, the remainder were scored using imaging studies that may overestimate/underestimate the extent of disease. In addition, only patients with squamous cell carcinoma were recruited in this study. In contrast, the incidence of adenocarcinoma is dramatically increasing in Western countries. Therefore, the results of this study may not be applicable to North American and European patients. Moreover, the use of neoadjuvant chemoradiotherapy 
has been an increasingly used treatment approach. The results of a multicenter phase III randomized trial (CROSS study) demonstrated that neoadjuvant chemoradiotherapy improved OS compared with surgery alone in patients with resectable (T2-3 N0-1 M0) esophageal or EGJ cancers. The median survival time was 49 months in the neoadjuvant chemoradiotherapy arm compared to 26 months in the surgery alone arm (24). Further development of the multidisciplinary management for patients with locally advanced esophageal cancer post-surgery using an adjuvant treatment as opposed to neoadjuvant chemoradiotherapy is warranted. The approach is currently being explored in China by investigators of the ZTOG1201 trial, a multicenter phase II trial of neoadjuvant and adjuvant chemoradiotherapy in locally advanced EC (NCT01463501).

In conclusion, this study demonstrated that data concerning the depth of primary tumor invasion and the number of lymph nodes involved may aid doctors in evaluating the recurrence risk of patients with TESCC that have been treated with curative surgery in a Chinese population. Further studies are required to clarify the correlation between recurrence and the different multidisciplinary treatment approaches.

\section{Acknowledgements}

This study was presented in part as a poster during the annual meeting of the 2011 European Multidisciplinary Cancer Congress (Abstract 6.558).

\section{References}

1. Guo M, Zhao YD, Yang HJ and Yan XF: Analysis of clinicopathological characteristics for 5406 cases of esophageal neoplasm. Chin J Cancer Prev Treat 15: 54-56, 2008.

2. Debevec L, Jeric T, Kovac V, et al: Is there any progress in routine management of lung cancer patients? A comparative analysis of an institution in 1996 and 2006. Radiol Oncol 43: 47-53, 2009.

3. Kovac V, Zwitter M and Zagar T: Population-based survey of malignant pleural mesothelioma in Slovenia: improved survival after introduction of chemotherapy. Radiol Oncol 46: 136-144, 2012.

4. Law SY, Fok M and Wong J: Pattern of recurrence after oesophageal resection for cancer: clinical implications. Br J Surg 83: 107-111, 1996.

5. Bhansali MS, Fujita H, Kakegawa T, et al: Pattern of recurrence after extended radical esophagectomy with three-field lymph node dissection for squamous cell carcinoma in the thoracic esophagus. World J Surg 21: 275-281, 1997.

6. Osugi H, Takemura M, Takada N, et al: Prognostic factors after esophagectomy and extended lymphadenectomy for squamous esophageal cancer. Br J Surg 89: 909-913, 2002.

7. Rice TW, Rusch VW, Ishwaran H, et al; Worldwide Esophageal Cancer Collaboration: Cancer of the esophagus and esophagogastric junction: data-driven staging for the seventh edition of the American Joint Committee on Cancer/International Union Against Cancer Staging Manuals. Cancer 116: 3763-3773, 2010.
8. Dresner SM and Griffin SM: Pattern of recurrence following radical esophagectomy with two-field lymphadenectomy. Br J Surg 87: 1426-1433, 2000.

9. Isono K, Sato H and Nakayama K: Results of a nationwide study on the three-field lymph node dissection of esophageal cancer. Oncology 48: 411-420, 1991.

10. Dixit S, Tilston M and Peter WM: Risk stratification for recurrence in patients with esophageal and junctional carcinoma treated with neoadjuvant chemotherapy and surgery. Med Oncol 27: 242-248, 2010.

11. Matsubara T, Ueda M, Nagao N, et al: Cervicothoracic approach for total mesesophageal dissection in cancer of the thoracic esophagus. J Am Coll Surg 187: 238-245, 1998.

12. Altorki NK and Skinner DB: Occult cervical nodal metastasis in esophageal cancer: preliminary results of three-field lymphadenectomy. J Thorac Cardiovasc Surg 113: 540-544, 1997.

13. Shiozaki H, Yano M, Tsujinaka T, et al: Lymph node metastasis along the recurrent nerve chain is indication for cervical lymph node dissection in thoracic esophageal cancer. Dis Esophagus 14: 191-196, 2001.

14. Chen J, Liu S, Pan J, et al: The pattern and prevalence of lymphatic spread in thoracic oesophageal squamous cell carcinoma. Eur J Cardiothorac Surg 36: 480-486, 2009.

15. Ando N, Iizuka T, Ide H, et al; Japan Clinical Oncology Group: Surgery plus chemotherapy compared with surgery alone for localized squamous cell carcinoma of the thoracic esophagus: a Japan Clinical Oncology Group Study - JCOG9204. J Clin Oncol 21: 4592-4596, 2003.

16. Pouliquen X, Levard H, Hay JM, et al: 5-Fluorouracil and cisplatin therapy after palliative surgical resection of squamous cell carcinoma of the esophagus. A multicenter randomized trial. French Associations for Surgical Research. Ann Surg 223: 127-133, 1996.

17. Lee J, Lee KE, Im YH, et al: Adjuvant chemotherapy with 5-fluorouracil and cisplatin in lymph node-positive thoracic esophageal squamous cell carcinoma. Ann Thorac Surg 80: $1170-1175,2005$

18. Heroor A, Fujita H, Sueyoshi S, et al: Adjuvant chemotherapy after radical resection of squamous cell carcinoma in the thoracic esophagus: who benefits? A retrospective study. Dig Surg 20: 229-235; discussion 236-237, 2003.

19. Shiozaki A, Yamagishi H, Itoi H, et al: Long-term administration of low-dose cisplatin plus 5-fluorouracil prolongs the postoperative survival of patients with esophageal cancer. Oncol Rep 13: 667-672, 2005.

20. Bystricky B, Okines AF and Cunningham D: Optimal therapeutic strategies for resectable oesophageal or oesophagogastric junction cancer. Drugs 71: 541-555, 2011.

21. Zhang J, Chen HQ, Zhang YW, et al: Adjuvant chemotherapy in oesophageal cancer: a meta-analysis and experience from the Shanghai Cancer Hospital. J Int Med Res 36: 875-882, 2008.

22. Lu JC, Qian PD, Zha WW, et al: The meta-analysis of randomized controlled trial of prophylactic radiotherapy for esophageal carcinoma after curative resection. J Evid Based Med 5: 166-171, 2005.

23. Macdonald JS, Smalley SR, Benedetti J, et al: Chemoradiotherapy after surgery compared with surgery alone for adenocarcinoma of the stomach or gastroesophageal junction. N Engl J Med 345: 725-730, 2001.

24. Gaast AV, van Hagen P, Hulshof M, et al: Esophagogastric junction cancer: Results from a multicenter randomized phase III study. J Clin Oncol (Meeting Abstracts) 28: 4004, 2010. 